\title{
O TRABALHO DE ENFERMAGEM EM LABORATÓRIOS DE ANÁLISES CLÍNICAS
}

Adriana Marques da Silva ${ }^{1}$

Marina Peduzzi?

Silva AM, Peduzzi M. O trabalho de enfermagem em laboratórios de análises clínicas. Rev Latino-am Enfermagem 2005 janeiro-fevereiro; 13(1):6571.

O artigo visa a caracterização do trabalho da enfermagem no setor de coleta de exames do laboratório de análises clínicas, identificando as atividades desempenhadas pelos diferentes agentes e sua inserção na estrutura organizacional. O referencial teórico está pautado nos estudos do processo de trabalho e de recursos humanos em saúde e enfermagem. Os dados foram coletados por meio de questionário, aplicado em uma amostra de 45 instituições e analisados quanti-qualitativamente. Os resultados mostram que, dentre os laboratórios estudados, 77,8\% têm auxiliares de enfermagem, $13 \%$ técnicos de enfermagem e 33,3\% enfermeiros. Observa-se a divisão do trabalho, na qual os enfermeiros desenvolvem sobretudo atividades gerenciais e os auxiliares e técnicos de enfermagem atividades assistenciais, não havendo diferenças no trabalho desses últimos. Há outros profissionais compartilhando das mesmas atividades realizadas pela enfermagem, a qual está subordinada a outras áreas de atuação em 24 (53,3\%) laboratórios. Evidencia-se a ausência de especificidade das ações de enfermagem e sua escassa autonomia no cenário estudado.

DESCRITORES: coleta de amostras; trabalho; enfermagem; força de trabalho

\section{NURSING WORK AT CLINICAL LABORATORIES}

The paper aims to show the features of the roles played by the nursing team in the sample collection service of a clinical laboratory by identifying the activities carried out by different agents and their insertion in the organizational structure. The theoretical reference framework is based on studies about the work process and human resources in health and nursing. Data were collected by means of a questionnaire, which was applied to a sample of 45 institutions. Data were analyzed quantitatively and qualitatively. Results show that $77.8 \%$ of the laboratories under analysis have nursing auxiliaries, $13 \%$ practical nurses and $33.3 \%$ nurses. Work division is observed: nurses are mainly in charge of managerial tasks, whereas nursing auxiliaries and nursing technicians are responsible for performing technical assistance activities, with no distinction between the latter's roles. There are other health professionals carrying out the same activities, and nursing is subordinated to other activity areas in 24 (53.3\%) laboratories. This study highlights the lack of specificity of nursing actions and their lack of autonomy in the study environment.

DESCRIPTORS: sample collection; work; nursing; labor force

\section{EL TRABAJO DE ENFERMERÍA EN LABORATORIOS DE ANÁLISIS CLÍNICOS}

El artículo tiene por objetivo caracterizar el trabajo de enfermería en el sector de colecta de exámenes de laboratorio de análisis clínicos, identificando las actividades desempeñadas por los diferentes agentes y su inserción en la estructura organizacional. El referencial teórico se basa en los estudios del proceso de trabajo y de recursos humanos en salud y enfermería. Los datos fueron recopilados mediante un cuestionario, aplicado en una muestra de 45 instituciones y analizado cuanti-cualitativamente. Los resultados muestran que, entre los laboratorios estudiados, el 77,8\% tiene auxiliares de enfermería, el 13\% técnicos de enfermería y el 33,3\% enfermeros. Se observa la división del trabajo, en la cual los enfermeros desarrollan principalmente actividades de gerencia y los auxiliares y técnicos de enfermería actividades asistenciales, sin que haya diferencias en el trabajo de esos últimos. Hay otros profesionales compartiendo las mismas actividades realizadas por la enfermería, la cual está subordinada a otras áreas de actuación en 24 (53,3\%) laboratorios. Queda de manifiesto la ausencia de especificidad de las acciones de enfermería y su escasa autonomía en el escenario estudiado.

DESCRIPTORES: toma de muestras; trabajo; enfermería; fuerza de trabajo

\footnotetext{
${ }^{1}$ Enfermeira, Mestre em Enfermagem em Administração, e-mail: enfadriana @ig.com.br; ${ }^{2}$ Enfermeira, Professor Doutor, e-mail: marinape @usp.br. Escola de Enfermagem da Universidade de São Paulo
} 


\section{INTRODUÇÃO}

A coleta de exames laboratoriais de pacientes em regime de internação e em situação ambulatorial nos laboratórios de análises clínicas é uma atividade que a enfermagem desenvolve e que contribui para a promoção, manutenção e recuperação da saúde. Nesse setor, a atuação da enfermagem está assegurada pela Portaria № CVS-01/ 2000 do Centro de Vigilância Sanitária, que trata das condições de funcionamento dos laboratórios de análises clínicas, patologia clínica e congêneres ${ }^{(1)}$ e pela Resolução COFEN - 146/1992 que preconiza a presença e responsabilidade de um enfermeiro nos locais onde existem ações de enfermagem sendo executadas ${ }^{(2)}$.

A sofisticação tecnológica laboratorial exige profissionais especializados nessa área, tanto na análise propriamente dita, como na obtenção da matéria-prima do laboratório, a amostra biológica, pois mesmo os equipamentos de última geração não conseguirão minimizar os interferentes ocasionados por coleta não adequada ao tipo de exame solicitado ${ }^{(3)}$. A obtenção de uma amostra biológica de boa qualidade exige do profissional um conhecimento específico sobre os tipos de análise e as diferentes etapas que compõem o processo de análises clínicas.

Frente aos avanços tecnológicos e à necessidade de contínuo aperfeiçoamento dos profissionais que atuam na área, realizou-se revisão da literatura sobre exames laboratoriais e enfermagem, nas bases de dados: BDENF, DEDALUS, LILACS, AdSaúde, SciELO e MEDLINE, na Biblioteca Virtual em Saúde (BIREME/OPAS/OMS) que evidenciou escassa literatura nacional e internacional sobre o tema. 0 levantamento bibliográfico identificou uma produção acerca dos aspectos ligados à saúde do trabalhador, biossegurança e sistemas de qualidade nos laboratórios. Observaram-se raras referências especificamente ao setor de coleta e a ausência de discriminação das categorias de trabalhadores que compõem essa área de atuação. Nas publicações que contemplam os agentes da enfermagem, esses aparecem sobretudo relacionados à execução do procedimento técnico e aos cuidados antes e depois da coleta. Também identificou-se a predominância da abordagem clínica dos exames sob a forma de manuais ${ }^{(4)}$.

Considerando a escassa produção teórica sobre o processo de trabalho de enfermagem nesse setor e, partindo-se do pressuposto que a coleta de exames contempla a execução de ações de enfermagem, foi desenvolvida investigação com o objetivo de caracterizar o trabalho da enfermagem no setor de coleta de exames do laboratório de análises clínicas, identificando as atividades desempenhadas pelos diferentes agentes e sua inserção na estrutura organizacional.

\section{REFERENCIAL TEÓRICO}

O referencial teórico adotado advém dos estudos do processo de trabalho em saúde e em enfermagem, destacando-se a categoria divisão do trabalho e dos estudos sobre recursos humanos em saúde.

O conceito de processo de trabalho foi desenvolvido por Marx em sua obra intitulada O Capital, em que define o trabalho como um processo, no qual os seres humanos atuam sobre as forças da natureza, submetendo-as ao seu controle e transformando os recursos naturais em bens e serviços úteis à vida humana, social e individual. Ao modificar a natureza, o trabalhador aciona suas energias físicomusculares e mentais ${ }^{(5)}$. O trabalho constitui, então, mediação entre 0 homem e a natureza, operando transformações requeridas pelas necessidades humanas ${ }^{(6)}$.

$\mathrm{Na}$ área da saúde, as pesquisas sobre o trabalho iniciaramse na América Latina e no Brasil, respectivamente, nos anos 60 e 70 do século XX. Esses estudos realizam uma interlocução com os conceitos marxistas de trabalho, buscando melhor compreensão das dimensões e elementos do processo de trabalho em saúde, considerando a historicidade das práticas. $\mathrm{Na}$ enfermagem, a introdução dessa abordagem teórico-metodológica iniciou-se nos anos 80 do século XX, com os estudos sobre o saber da enfermagem ${ }^{(7)}$.

O processo de trabalho tem como elementos constituintes a atividade adequada a um fim, que é o trabalho propriamente dito; 0 objeto de trabalho, que é a matéria a ser transformada, que pode estar em seu estado bruto ou ser resultante de um trabalho prévio, como as matérias-primas; e os meios de trabalho, que são os instrumentos utilizados pelo trabalhador ${ }^{(5-6,8)}$.

Os agentes constituem um dos elementos do processo de trabalho e, por isso, devem ser analisados no interior das relações recíprocas entre objeto, instrumentos e atividades, bem como no interior do processo de divisão do trabalho ${ }^{(9)}$.

Por meio da ação do agente, é possível observar e expressar a existência concreta, mas, nem sempre evidente, de uma dinâmica entre objetos, saberes, instrumentos materiais e atividades do processo de trabalho. Esse agente não é apenas o instrumento de viabilização concreta desse processo, inserindo ferramentas entre ele próprio e o objeto com uma dada finalidade e colocando-se como portador de um projeto específico, mas, também, é sujeito, conforme traz para dentro do trabalho outros projetos de caracteres coletivos e pessoais $^{(6)}$.

No campo da enfermagem, os objetos de trabalho são 0 cuidado de enfermagem e seu gerenciamento. Cuidado esse concebido como um conjunto de ações de acompanhamento contínuo do usuário, ou população, no transcorrer das doenças, ou ao longo de processos sociovitais (saúde da criança, do adolescente, da mulher, do idoso, entre outros), visando a promoção, prevenção e recuperação da saúde ${ }^{(9)}$.

Outro aspecto importante é sua divisão técnica e social que advém de um processo historicamente construído. A divisão técnica do trabalho consiste no parcelamento dos processos que determinam as 
várias etapas de produção de um dado produto, executadas por distintos trabalhadores. Essa modalidade de divisão tem como característica central o fato do trabalhador parcial não produzir nenhum produto por completo isoladamente, sendo necessária a participação de vários trabalhadores especializados ${ }^{(6)}$.

Na enfermagem, também, ocorre a divisão técnica e social do trabalho, tanto externa, quando divide o espaço de atuação com o médico e outros profissionais de saúde, como internamente, pelo parcelamento do cuidado e do aparecimento de distintas categorias de trabalhadores de enfermagem.

No Brasil, as ações de cuidado de enfermagem são divididas entre múltiplos agentes: auxiliar de enfermagem, técnico de enfermagem, enfermeiro e trabalhadores de enfermagem sem qualificação técnica formal e regular. $\mathrm{O}$ enfermeiro assume as atividades mais intelectuais de ensino e gerenciamento do cuidado e/ou dos serviços, enquanto 0 pessoal de enfermagem de nível médio, cujo trabalho possui caráter mais manual, responde pela maioria das atividades assistenciais ${ }^{(6,9-10)}$.

\section{METODOLOGIA}

Esta pesquisa foi desenvolvida nas modalidades qualitativa e quantitativa. A análise quantitativa contemplou o tratamento estatístico dos dados armazenados em banco de dados, seguido da análise qualitativa com base no referencial teórico.

O campo de estudo foi constituído por laboratórios de análises clínicas públicos e privados e unidades básicas de saúde que possuíam o setor de coleta de material biológico, no Município de São Paulo, em 2002, sendo esse o critério de inclusão, e o de exclusão, aqueles que não o fossem ou não tivessem o setor de coleta de amostras.

Inicialmente a amostra foi definida baseada nas listagens fornecidas pela Fundação Sistema Estadual de Análise de Dados e pela Sociedade Brasileira de Análises Clínicas que forneceram relações com 138 e 584 instituições, respectivamente.

Desses 722 serviços, foi feita uma primeira depuração considerando o critério de exclusão indicado nas razões sociais e/ou nomes fantasias dos serviços, resultando em 595 instituições. A amostra foi definida pelo método de amostragem sistemática não-probabilística com o intervalo determinado pelo sorteio do número três na tabela de dígitos aleatórios.

Vale a pena ressaltar que a listagem geral estava incompleta e desatualizada quanto às informações. Para completar os dados, foi consultado o guia de assinantes no endereço eletrônico www.telefonica.com.br*.

Um primeiro contato telefônico foi feito a fim de confirmar dados da listagem e o critério de inclusão. Desse processo, 180 (30,3\%) laboratórios foram selecionados e 415 (69,7\%) excluídos. A alta taxa de exclusão deu-se, sobretudo, pela dificuldade para conseguir informações corretas, pois os telefones eram inexistentes na listagem geral e não se conseguiu localizá-los no site acima referido. Dada essa dificuldade, foi feito contato com a Vigilância Sanitária de São Paulo que também forneceu outra listagem composta por 44 laboratórios de análises clínicas e, desses, 16 serviços foram agregados à listagem existente, compondo uma população de 196 instituições.

Considerando-se a expressiva perda amostral, optou-se por desenvolver a pesquisa de campo com a totalidade dos laboratórios identificados que atenderam aos critérios de inclusão.

Os dados empíricos foram coletados por meio de um questionário, em especial, pela facilidade de aplicação simultânea a todos os elementos da população ${ }^{(11)}$. 0 instrumento contemplou informações relacionadas à caracterização da instituição, dos recursos humanos atuantes no setor de coleta e das atividades desenvolvidas por esses agentes.

O projeto de pesquisa foi aprovado pelo Comitê de Ética em Pesquisa da Escola de Enfermagem da Universidade de São Paulo, procedendo-se, então, ao teste piloto do instrumento de coleta de dados em dois laboratórios clínicos, um hospitalar e outro ambulatorial.

A fase de coleta durou cinco meses e foi realizada no período de abril a setembro de 2003. Da população de 196 laboratórios, apenas $16(8 \%)$ retornaram o questionário, assim, foi feita uma busca ativa por telefone que resultou em mais 28 instituições excluídas, restando 168 laboratórios válidos para pesquisa. Do total, retornaram mais 29 , totalizando $45(26,8 \%)$ questionários respondidos. Assim, obteve-se uma amostragem caracterizada como amostra por conveniência ${ }^{* \star}$, pois participaram da pesquisa os laboratórios disponíveis, ou seja, que responderam ao instrumento de coleta de dados.

As informações coletadas foram armazenadas em um banco de dados e submetidas a tratamento estatístico por meio do programa Intercooled Estata 7.0. Os aspectos qualitativos foram analisados com base no referencial teórico adotado, particularmente, as categorias analíticas do processo de trabalho, divisão do trabalho e recursos humanos em saúde.

\section{RESULTADOS}

Os resultados relativos à caracterização dos recursos humanos apontam que o profissional predominante é 0 auxiliar de enfermagem, visto que é referido em $35(77,8 \%)$ laboratórios estudados.

\footnotetext{
*Telefônica Telecomunicações de São Paulo SA. Guia de assinantes on-line. São Paulo; 2002. Disponível em <http://www.telefonica.com.br> (15 dez 2002)

** Triola MF. Introdução à estatística. $7^{a}$ ed. Rio de Janeiro (RJ): LTC; 1999. Introdução à estatística, p. 1-16
} 
No que se refere ao pessoal auxiliar, aparecem, em seguida, o auxiliar de laboratório em 19 (42,2\%) instituições e o auxiliar de patologia clínica, em 5 (11\%).

No nível técnico, a categoria profissional mais freqüente é 0 técnico de laboratório, em $16(35,6 \%)$ serviços, seguido pelo técnico de patologia clínica em $8(17,8 \%)$ e pelo técnico de enfermagem em 6 $(13,3 \%)$ laboratórios. O profissional de nível superior mais freqüente é o biomédico que aparece em $23(51,1 \%)$ laboratórios, seguido pelo enfermeiro em 15 (33,3\%) e pelo biólogo em 14 (31,1\%) instituições.

0 médico e o farmacêutico-bioquímico foram mencionados em $12(26,7 \%)$ serviços. Convém salientar que $3(6,7 \%)$ laboratórios têm em seu quadro de coleta de exames outros trabalhadores sem qualificação técnica formal e regular para o exercício desse trabalho.

Quanto à caracterização das atividades desenvolvidas no setor de coleta de exames, os resultados são apresentados, distinguindo as atividades de assistência e de gerenciamento. Apesar da atividade de treinamento em serviço ser, usualmente, considerada de natureza gerencial, neste estudo, será apresentada separadamente, visto ter sido identificado que não ocorre em 7 (15,6\%) laboratórios (Tabela 1).

Considerados os 45 laboratórios estudados, observa-se que as atividades gerenciais do enfermeiro varia de $9(20 \%)$ a $17(37,8 \%)$ serviços e as atividades assistenciais variam de $2(4,4 \%)$ a $12(26,7 \%)$ laboratórios, sendo o treinamento em serviço executado por esse profissional em $16(35,6 \%)$ instituições. Para o técnico de enfermagem, as atividades assistenciais variam de $1(2,2 \%)$ a $9(20 \%)$ serviços e as gerências variam de $1(2,2 \%)$ a $2(4,4 \%)$ laboratórios, o treinamento é realizado por esse agente em $2(4,4 \%)$ instituições. 0 auxiliar de enfermagem, por sua vez, executa sobretudo atividades assistenciais, pois essas variam de $3(6,7 \%)$ a $35(77,8 \%)$ laboratórios e as gerências variam de $1(2,2 \%)$ a $9(20 \%)$ serviços, o treinamento é realizado por esse trabalhador em $3(6,7 \%)$ instituições.

No que se refere, particularmente, à divisão de trabalho nas dimensões assistencial e gerencial nas 15 instituições que possuem enfermeiros, $3(20 \%)$ informaram não haver divisão das atividades entre esses e, dentre os 35 laboratórios que contam com auxiliares de enfermagem em seu quadro, 19 (54,3\%) referiram não constar distinção na categoria. Nos 6 serviços que informam a existência de técnicos ocorre a divisão de atividades.

Nos laboratórios que informaram a existência de divisão de atividades, as ações exclusivamente assistenciais são praticadas pelo auxiliar de enfermagem em 14 (40\%) instituições, pelo técnico de enfermagem em $3(50 \%)$ serviços, enquanto 0 enfermeiro não desenvolve ações unicamente assistenciais. Esses realizam somente atividades gerenciais em 4 (26,7\%) instituições e o técnico em 1 (16,7\%) serviço. Nota-se que o enfermeiro realiza ambas as atividades, de assistência e de gerenciamento, em 8 (53,3\%) laboratórios, o técnico de enfermagem em $2(33,3 \%)$ e 0 auxiliar de enfermagem, em 2 $(5,7 \%)$ serviços.

Tabela 1 - Distribuição dos laboratórios estudados, segundo realização de atividades assistenciais e gerenciais, por categoria profissional do setor de coleta de exames. Município de São Paulo, 2003

\begin{tabular}{|c|c|c|c|c|c|c|c|c|c|c|c|c|c|c|c|c|c|}
\hline & \multirow{5}{*}{ Atividades desenvolvidas } & \multirow{3}{*}{\multicolumn{4}{|c|}{ Enfermeiro }} & \multirow{2}{*}{\multicolumn{4}{|c|}{$\begin{array}{l}\text { Técnico de } \\
\text { Enfermagem }\end{array}$}} & \multirow{3}{*}{\multicolumn{4}{|c|}{$\begin{array}{c}\text { Auxiliar de } \\
\text { Enfermagem } \\
\text { Laboratório }\end{array}$}} & \multirow{3}{*}{\multicolumn{4}{|c|}{$\begin{array}{c}\text { Outros Trabalhadores } \\
\text { Nível Superior } \\
\text { Laboratório }\end{array}$}} \\
\hline & & & & & & & & & & & & & & & & & \\
\hline & & & & & & \multicolumn{4}{|c|}{ Laboratório } & & & & & & & & \\
\hline & & \multicolumn{4}{|c|}{ Sim Não } & & im & & ão & \multicolumn{2}{|c|}{$\operatorname{Sim}$} & \multicolumn{2}{|c|}{ Não } & \multicolumn{2}{|c|}{ Sim } & \multicolumn{2}{|c|}{ Não } \\
\hline & & № & $\%$ & № & $\%$ & № & $\%$ & № & $\%$ & № & $\%$ & № & $\%$ & № & $\%$ & № & $\%$ \\
\hline \multirow{9}{*}{ 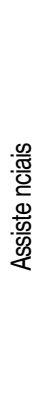 } & Recepção de amostras já coletadas & 5 & 11,1 & 40 & 88,9 & 4 & 8,9 & 41 & 91,1 & 22 & 48,9 & 23 & 51,1 & 14 & 31,1 & 31 & 68,9 \\
\hline & Coleta de exames, em geral (sangue, urina, fezes, culturas, micológicos, etc.) & 10 & 22,2 & 35 & 77,8 & 9 & 20 & 36 & 80 & 35 & 77,8 & 10 & 22,2 & 17 & 37,8 & 28 & 62,2 \\
\hline & Coleta de exames de maior complexidade (provas funcionais, teste do pezinho, etc.) & 7 & 15,6 & 38 & 84,4 & 2 & 4,4 & 43 & 95,6 & 13 & 28,9 & 32 & 71,1 & 10 & 22,2 & 35 & 77,8 \\
\hline & Auxílio em procedimentos médicos (mielograma, líquor, etc.) & 9 & 20 & 36 & 80 & 4 & 8,9 & 41 & 91,1 & 11 & 24,4 & 34 & 75,6 & 5 & 11,1 & 40 & 88,9 \\
\hline & Gasometria arterial & 11 & 24,4 & 34 & 75,6 & 1 & 2,2 & 44 & 97,8 & 3 & 6,7 & 42 & 93,3 & 9 & 20 & 36 & 80 \\
\hline & Manipulação de cateteres centrais & 9 & 20 & 36 & 80 & 1 & 2,2 & 44 & 97,8 & 4 & 8,9 & 41 & 91,1 & 4 & 8,9 & 41 & 91,1 \\
\hline & Orientações sobre os exames & 12 & 26,7 & 33 & 73,3 & 3 & 6,7 & 42 & 93,3 & 22 & 48,9 & 23 & 51,1 & 25 & 55,6 & 20 & 44,4 \\
\hline & Encaminhamento das amostras coletadas ao setor técnico & 2 & 4,4 & 43 & 95,6 & 4 & 8,9 & 41 & 91,1 & 24 & 53,3 & 21 & 46,7 & 11 & 24,4 & 34 & 75,6 \\
\hline & Coleta domiciliar & 2 & 4,4 & 43 & 95,6 & 2 & 4,4 & 43 & 95,6 & 10 & 22,2 & 35 & 77,8 & 5 & 11,1 & 40 & 88,9 \\
\hline \multirow{8}{*}{ 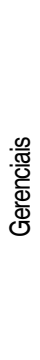 } & Teste de materiais utilizados na coleta de exames & 9 & 20 & 36 & 80 & 2 & 4,4 & 43 & 95,6 & 9 & 20 & 36 & 80 & 17 & 37,8 & 28 & 62,2 \\
\hline & Supervisão & 15 & 33,3 & 30 & 66,7 & 2 & 4,4 & 43 & 95,6 & 1 & 2,2 & 44 & 97,8 & 25 & 55,6 & 20 & 44,4 \\
\hline & Coordenação & 14 & 31,1 & 31 & 68,9 & 1 & 2,2 & 44 & 97,8 & 1 & 2,2 & 44 & 97,8 & 28 & 62,2 & 17 & 37,8 \\
\hline & Planejamento de ações de enfermagem & 17 & 37,8 & 28 & 62,2 & - & - & 45 & 100 & 1 & 2,2 & 44 & 97,8 & 11 & 24,4 & 34 & 75,6 \\
\hline & Participação na seleção de recursos humanos em enfermagem & 14 & 31,1 & 31 & 68,9 & - & - & 45 & 100 & 1 & 2,2 & 44 & 97,8 & 15 & 33,3 & 30 & 66,7 \\
\hline & Previsão e provisão de pessoal & 12 & 26,7 & 33 & 73,3 & - & - & 45 & 100 & - & - & 45 & 100 & 18 & 40 & 27 & 60 \\
\hline & Elaboração de escalas & 16 & 35,6 & 29 & 64,4 & - & - & 45 & 100 & 1 & 2,2 & 44 & 97,8 & 17 & 37,8 & 28 & 62,2 \\
\hline & Treinamento em serviço & 16 & 35,6 & 29 & 64,4 & 2 & 4,4 & 43 & 95,6 & 3 & 6,7 & 42 & 93,3 & 18 & 40 & 27 & 60 \\
\hline
\end{tabular}

- A distribuição das atividades considerou a freqüência em que foram referidas nos 45 laboratórios estudados 
Quanto à inserção da enfermagem na estrutura organizacional, os resultados mostram que está subordinada à chefia do próprio laboratório em $13(28,9 \%)$ instituições, seguida pela subordinação à direção do serviço em 11 (24,4\%) e à área de enfermagem em $8(17,8 \%)$ dos serviços.

\section{ANÁLISE DE DADOS}

Na coleta de exames nas instituições pesquisadas, o auxiliar de enfermagem é o profissional predominante. Entende-se que isso ocorre por ser mais acessível aos laboratórios, do ponto de vista econômico, visto que cabe ao auxiliar de enfermagem a execução de tarefas mais manuais da assistência de enfermagem.

A existência de outros trabalhadores sem qualificação técnica formal e regular, atuando no setor de coleta de exames, chama a atenção. Esses são apontados em 3 (6,7\%) instituições, no entanto, consideradas as ações desenvolvidas, o agentes aparecem em 13 $(35,1 \%)$ laboratórios, realizando atividades de recepção e encaminhamento das amostras coletadas.

A respeito da caracterização das atividades realizadas pelos recursos humanos de enfermagem, observa-se que o gerenciamento é realizado predominantemente pelo enfermeiro e, em menor freqüência, pelo técnico de enfermagem e auxiliar de enfermagem.

Nas atividades assistenciais, observa-se tendência oposta, ou seja, essas são executadas com maior freqüência pelos auxiliares de enfermagem e técnicos de enfermagem.

Portanto, os resultados desta pesquisa confirmam aqueles encontrados em outros estudos que apontam para o trabalho, majoritariamente, gerencial do enfermeiro e com predominância assistencial para o pessoal de enfermagem de nível médio ${ }^{(10,12)}$. A enfermeira com freqüência assume posição de gerente da assistência de enfermagem e da organização institucional, porque essa precisa de um agente que conheça a essência do trabalho de enfermagem, mas, não necessariamente para executá-lo. Assim, nota-se que o enfermeiro assume as atividades mais intelectuais, relacionadas ao planejamento, organização, supervisão, avaliação do cuidado e/ou dos serviços, enquanto o pessoal de enfermagem de nível médio, cujo trabalho possui caráter mais manual, responde pela maioria das atividades assistenciais e do cuidado de enfermagem ${ }^{(10)}$.

Outro aspecto relevante observado na análise dos resultados refere-se à presença marcante de ações de enfermagem no trabalho cotidiano, realizado nos setores de coleta de exames, tais como: punção venosa, orientações para o preparo de exames, manipulação de cateteres, sondagens, coleta de secreções e realização de curativos em feridas, após a coleta de material biológico e outras. Mas tais atividades também são executadas por trabalhadores de nível médio e superior de outras áreas de atuação.

Esse aspecto da análise está relacionado com o dilema da regulação profissional na área da saúde. Na atualidade, vem ocorrendo a introdução de um novo paradigma para a regulamentação das profissões, especialmente, na área da saúde, baseado na proteção do público contra efeitos prejudiciais da prática das profissões, ou seja, deve prevalecer a defesa dos interesses públicos no sentido da ampliação do acesso, eficiência econômica, plena utilização das potencialidades e competências dos recursos humanos em um ambiente em que a proteção e o bem-estar da clientela devem ser constantemente avaliados e assegurados $^{(13)}$. Ou seja, esse novo paradigma prioriza a defesa dos interesses públicos e não dos interesses das corporações profissionais.

No setor de coleta de exames dos laboratórios, as ações desenvolvidas pelos agentes de nível superior são compartilhadas entre a enfermagem, biomedicina, farmácia-bioquímica, biologia e medicina. Por outro lado, as ações realizadas pelos trabalhadores de nível médio são divididas entre os auxiliares e técnicos de enfermagem, patologia clínica e de laboratório. Assim sendo, o compartilhamento das atividades de coleta de exames entre agentes de enfermagem e trabalhadores de outras áreas profissionais deve ser analisado na perspectiva dos interesses dos usuários do serviço, isto é, buscando evitar a exposição da clientela a riscos e potenciais danos à saúde, acarretados pela execução dessas ações por trabalhadores sem a necessária qualificação técnica e não apenas da perspectiva corporativa.

Outra realidade dos laboratórios de análises clínicas é que a tecnologia de ponta está concentrada no setor técnico, ou seja, no setor de análise da amostra, no qual foi possível a incorporação da microeletrônica e da automação, determinando o grau de modernidade e o reconhecimento social que os laboratórios possuem, o que vem acompanhado da necessidade de um alto grau de qualificação técnicocientífica dos respectivos profissionais. Já no setor de coleta de exames, executa-se um trabalho centrado em atividades de caráter manual, de modo que esse tende a ter menor valorização técnica e social por referência ao setor de análises, bem como a concentrar maior número de trabalhadores de nível médio.

Nesse sentido, vale destacar que, no processo de divisão do trabalho, não apenas a divisão técnica e pormenorizada de atividades é reproduzida, mas, também, a desigual valoração social desses trabalhos parcelares ou especializados.

Outro ponto a ser ressaltado é o fato de que o técnico de enfermagem e o auxiliar de enfermagem, no setor de coleta de exames, desenvolvem basicamente as mesmas atividades, sem distinção no trabalho executado por ambos. Esse resultado também é confirmado 
por outros estudos que assinalam a ausência de diferenças no trabalho desenvolvido pelos auxiliares e técnicos de enfermagem ${ }^{(14-15)}$, revelando um subaproveitamento das potencialidades do técnico de enfermagem.

Esse contexto permite analisar que, apesar da Lei do Exercício Profissional de Enfermagem fazer distinção das diferentes categorias, na prática, a legislação não é validada em sua totalidade, visto que o técnico e o auxiliar de enfermagem executam as mesmas ações nos laboratórios de análises clínicas.

Nesta pesquisa, os resultados encontrados mostram distorção das atividades previstas para cada categoria profissional, pois, nos laboratórios estudados, a atividade de gasometria arterial, que é privativa do enfermeiro, é executada pelo técnico de enfermagem em $1(2,2 \%)$ serviço e pelo auxiliar de enfermagem em $3(6,7 \%)$ instituições. Encontra-se, também, o auxiliar de enfermagem realizando atividades de supervisão, coordenação e planejamento das ações de enfermagem em 1 (2,2\%) laboratório pesquisado.

A análise da inserção da enfermagem na estrutura organizacional mostra que essa encontra-se majoritariamente subordinada à chefia do próprio laboratório ou à direção do serviço, evidenciando que a enfermagem está subordinada a outras áreas de trabalho, o que lhe confere escassa autonomia quanto à sua inserção institucional. Esse resultado é consistente e coerente com outros aspectos analisados que dizem respeito à indiferenciação entre ações de enfermagem e profissionais de outras áreas, ausência de diferenças entre os agentes da própria enfermagem, especialmente, entre o auxiliar e o técnico de enfermagem, apontando para a pouca visibilidade e valorização social da prática de enfermagem.

\section{CONSIDERAÇÕES FINAIS}

$\mathrm{Na}$ caracterização das atividades desenvolvidas pelos trabalhadores de enfermagem no setor de coleta de exames, esta pesquisa confirma as evidências de outros estudos ${ }^{(8-10,12,15)}$ à medida que mostra divisão de trabalho inequívoca entre as três categorias profissionais que compõem a força de trabalho da área de enfermagem.

Os auxiliares de enfermagem executam quase, exclusivamente, ações assistenciais. Para os técnicos de enfermagem também predominam as atividades assistenciais, embora os informantes tenham apontado a divisão do trabalho assistencial e gerencial na equipe de enfermagem, e o resultado assinale ambas as modalidades, de forma mais eqüitativa para o técnico. Por outro lado, os enfermeiros concentram seu trabalho na dimensão gerencial.

Nota-se uma situação de trabalho na qual os auxiliares executam o cuidado direto na coleta de exames, o enfermeiro gerencia o processo e os técnicos de enfermagem participam de ambos os momentos, tanto na execução como na coordenação do trabalho.

Além disso, observa-se que outros profissionais de nível superior respondem integralmente, ou participam do gerenciamento da equipe de enfermagem, o que contraria as determinações da regulação profissional da área que prevê a coordenação do trabalho de enfermagem sob a responsabilidade do enfermeiro. Se, por um lado, há agentes da enfermagem respondendo a outros profissionais não enfermeiros, por outro, os laboratórios de pequeno porte com baixa demanda alegam não possuírem condições financeiras para manter um enfermeiro contratado para responder pelo pessoal de enfermagem no setor de coleta, revelando que a realidade de alguns laboratórios de análises clínicas não permite o cumprimento das normas de regulação profissional estabelecidas.

A ausência de diferenças entre 0 trabalho executado pelo auxiliar e pelo técnico de enfermagem também foi identificada, à exceção de uma maior responsabilização do último por ações gerenciais.

Fica evidente, portanto, a necessidade de amplo debate sobre a regulação do trabalho em saúde e em enfermagem, bem como do trabalho no setor de coleta de exames de análises clínicas. Esse debate deve ser travado, não no sentido de buscar estabelecer fronteiras rígidas entre os diversos trabalhos especializados, mas para garantir maior clareza quanto à esfera de responsabilidade de cada área profissional, atendendo, simultaneamente, os esquemas dinâmicos e flexíveis de atribuições no mercado de trabalho atual, particularmente, dos trabalhadores de saúde de nível médio que apresentam um leque muito diversificado de denominações.

Outro aspecto que merece ampla discussão, no campo da saúde e da enfermagem, diz respeito à representação social e à efetiva inserção dos trabalhadores de enfermagem nas práticas de saúde, visto que esta pesquisa, assim como outros estudos, demonstra que 0 trabalho de enfermagem ainda apresenta pouca visibilidade e valor na divisão social do trabalho. Esses agentes ocupam-se de atividades que respondem à necessidade social de cuidados e, nesse sentido, são imprescindíveis ao bem-estar e ao enfrentamento do sofrimento, da dor e dos agravos à saúde, mas são pouco reconhecidos socialmente.

Por fim, destacam-se os limites desta investigação no tratamento e na compreensão dos dois últimos aspectos acima referidos, ou seja, da regulação profissional e da valorização do trabalho de enfermagem, pois o objetivo do estudo foi caracterizar o processo de trabalho no setor de coleta especificamente quanto às atividades da enfermagem e sua inserção na estrutura. Contudo, o referencial teórico do processo de trabalho na vertente da dialética marxista permite evidenciar dimensões mais gerais relacionadas ao trabalho, à medida que pressupõe o princípio da contradição, totalidade e historicidade. 


\section{REFERÊNCIAS BIBLIOGRÁFICAS}

1. Portaria $n^{\circ}$ CVS-01, 18.01.00. Dispõe sobre as condições de funcionamento dos laboratórios de análises clínicas, patologia clínica e congêneres. Diário Oficial Estado de São Paulo, São Paulo, 19 fev 2000. Seção 1, p.1-63.

2. Resolução COFEN-146, 01.06.92. Dispõe sobre a obrigatoriedade de haver enfermeiro em todas as unidades de serviço em que são desenvolvidas ações de enfermagem. Rio de Janeiro (RJ): Conselho Federal de Enfermagem. 1992.

3. Moura RAA. Colheita de material para exames de laboratório: assegurando a qualidade dos serviços no laboratório clínico. São Paulo (SP): Atheneu; 1998.

4. Malarkey LM, McMorrow ME. Nurse's manual of laboratory tests and diagnostic procedures. Philadelphia: WB. Saunders; 1996.

5. Liedke ER. Trabalho. In: Cattani AD, organizador. Trabalho e tecnologia: dicionário crítico. Petrópolis (RJ): Vozes; 1997. p. 269-74. 6. Peduzzi M. Equipe multiprofissional de saúde: a interface entre trabalho e interação. [Tese]. Campinas (SP): Faculdade de Ciências Médicas/ UNICAMP; 1998.

7. Almeida MCP, Mishima SM, Peduzzi M. A pesquisa em enfermagem fundamentada no processo de trabalho: em busca da compreensão e qualificação da prática de enfermagem. Anais do $51^{\circ}$ Congresso Brasileiro de Enfermagem; 1999 outubro 2-7; Florianópolis; Santa Catarina: ABEn - Seção SC; 2000. p. 259-77.

8. Almeida MCP, Rocha SMM. Considerações sobre a enfermagem enquanto trabalho. In: Almeida MCP, Rocha SMM, organizadoras. 0 trabalho de enfermagem. São Paulo (SP): Cortez; 1997. p.15-26.

9. Peduzzi M, Anselmi ML. O processo de trabalho de enfermagem: a cisão entre o planejamento e execução do cuidado. Rev Bras Enfermagem julho/agosto 2002; 55(4):392-8.

10. Almeida MCP; Rocha JSY. O saber de enfermagem e sua dimensão prática. São Paulo (SP): Cortez; 1986.

11. Cassiani SHB. A coleta de dados nas pesquisas em enfermagem: estratégias, validade e confiabilidade. [Dissertação]. Ribeirão Preto (SP): Escola de Enfermagem de Ribeirão Preto/USP; 1987.

12. Castellanos BEP. $O$ trabalho do enfermeiro: a procura e o encontro de um caminho para o seu estudo: da abordagem mecânico-funcionalista à pesquisa emancipatória. [Tese]. São Paulo (SP): Escola de Enfermagem/USP; 1987.

13. Girardi SN, Seixas PH. Dilemas da regulamentação profissional na área da saúde: questões para um governo democrático e inclusionista. Formação maio 2002; 1(5):29-43.

14. Ministério da Saúde (BR). Perfil de ações do técnico de enfermagem no Brasil. Brasília (DF): Ministério da Saúde; 2003.

15. Peduzzi M, Anselmi ML. O processo de trabalho do auxiliar de enfermagem. Formação janeiro 2003; 3(7):73-87. 